\title{
Live Streaming Motion Detection Camera Security System with Email Notification using Raspberry Pi
}

\author{
Angela Antony ${ }^{1}$, Prof. G. R. Gidveer ${ }^{2}$ \\ ${ }^{1,2}$ Dept. Electronics and Telecommunication, MGM's Jawaharlal Nehru Engineering College, Aurangabad, \\ India) \\ ('angelaantony3@gmail.com, ${ }^{2}$ grgidveer@yahoo.com)
}

\begin{abstract}
In the present day scenario the security forms the most important part of our lives. Let it be in any form of life. Every one of us is in some way or the other bothered about the security of the house or the image or the near and dear ones. Now as the need for a very economic system has arise the solution has been found in a camera based security system based on the Raspberry Pi software that is extremely user friendly and cost effective. It is a credit card size computer that uses its own camera board for the security system. It is accompanied with motion detection software that enables the camera to detect the motion and save the clicked image and immediately fwd to the portable devices for live streaming. For email notification a python script is used. With these components, a cost effective and efficient security camera system is made and reported here.
\end{abstract}

Keywords: email, live streaming, motion detecting, notifications, Raspberry Pi, python.

\section{INTRODUCTION}

In this new era of technology the race is to provide a user friendly device which is cost effective. The capability of the device should be of such a nature that the cost of the item seems to be always less. Such a device is offered by Raspberry Pi systems. Its capability ranges from being a security system to a VPN server. Unlike other computers it has the capability to accept several program which includes "Python" language. Generally a security system will cost an approx of Rs 6000 to Rs 8000 where as the Raspberry Pi system will at max cost only Rs 4000. so who will be interested in buying a security system of Rs 6000 (approx) where in one can get a system in a price range of Rs 2000 and with added features of email notification.

Apart from the price tag the next aspect is the user friendly nature of the system. As we are all aware that in India not everyone is a tech savvy individual. There by making it more important to ensure that the user does has to rewind the entire system to view the problem. In this system the email notification feature helps the user to see what is wrong than to see the entire video to find the error. The Raspberry Pi system is not only user friendly it also enables a individual wit medium knowledge to assemble the system if the necessary raw material is available and by creation of some extra files to support the operating system to store the data. So they are not only a money saving project but also a efficient security system.

\section{II.PROPOSED SYSTEM}

The proposed system has been designed to overcome the drawbacks of the previous security system and to improve the security, flexibility, efficiency whenever needed, having a security camera system may sometimes be impossible due to the exhaustive costs incurred during installation. The Raspberry Pi is a computer of a size of a credit card that has the capability to become a camera security system when its own camera board is used. It contains all the essential software to include motion detection which enables the Raspberry Pi's camera to detect motion and save the image as well as view a live streaming of the location from the camera. A python script, then directs the Raspberry Pi to send email notifications every time a motion is detected. With these components, a cost effective and efficient security camera system is made as reported here. The proposed system block diagram is shown in Figure 1.

2.1 COMPONENT DESCRIPTION

The proposed system consists of following major components.

- Raspberry Pi

- LAN Cable 
- HDMI Display

- PIR sensor

- Buzzer

- Keyboard and mouse

- Webcam

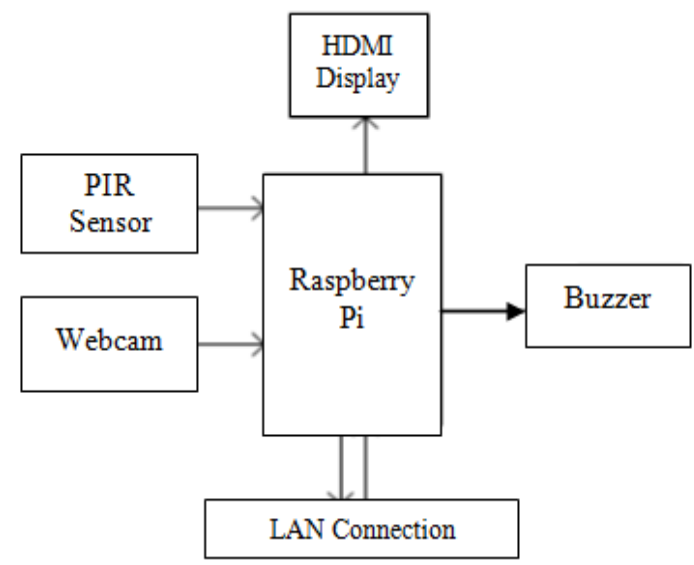

Fig.1: Block diagram of proposed system

\subsection{RASPBERRY PI}

An UK based company "Raspberry Pi Foundation" has developed a credit card sized single board computer which is called the Raspberry Pi System. The Raspberry Pi has a Broadcom BCM2835 System on a Chip (SoC), which includes an ARM1176JZF-S $700 \mathrm{MHz}$ processor. It has an internal storage of $512 \mathrm{MB}$, external storage supported up to $32 \mathrm{~GB}$, one Ethernet port, four-2.0 USB ports, one micro SD card slot, DSI display connector, one HDMI out port, one CSI Camera connector, 5V USB power, RCA video and audio jack as shown in the below figure.

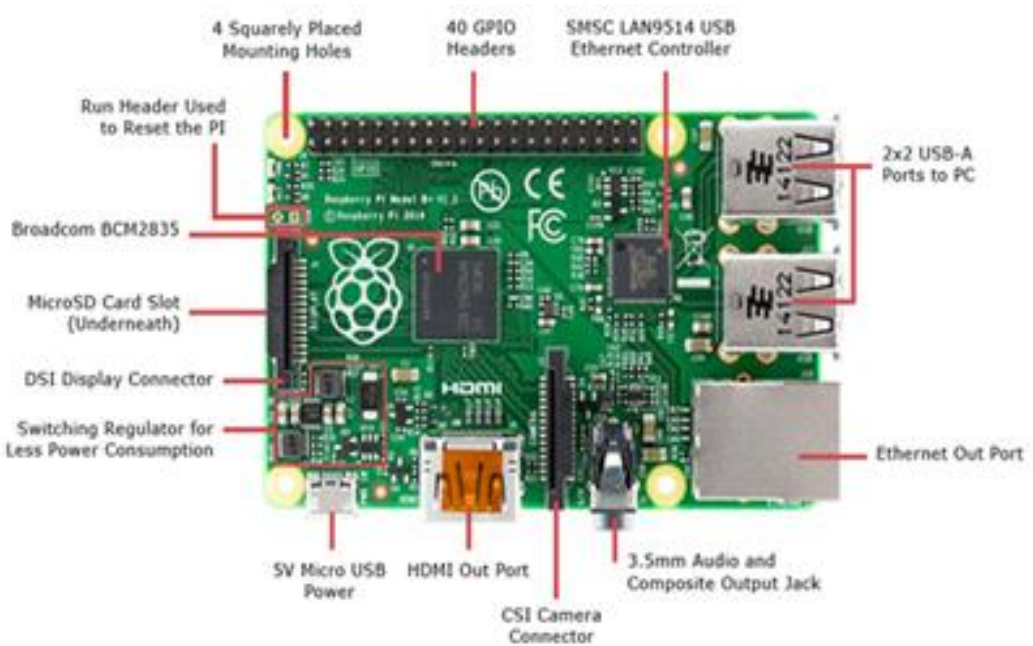

Fig.2: Raspberry Pi B+ Model

\subsection{PIN CONFIGURATION}

The Raspberry Pi B+ model CPU is of ARM 11 family, operates at 700MHz. The GPU having Broadcom Video core-IV, open GLES 2.0, 1080p30, H.264/MPEG-4, AVC high profile decoder and capable of $1 \mathrm{Gpixel} / \mathrm{s}, 1.5 \mathrm{G} \mathrm{Texel} / \mathrm{s}$ (or) $24 \mathrm{GFLOPs}$ of general-purpose (GPIO) compute. It operates $1 \mathrm{~mA}$ at $5 \mathrm{~V}$ power supply. With the help of GPIO pins we can control the devices sitting at home. The GPIO pin configuration is shown in below figure. 


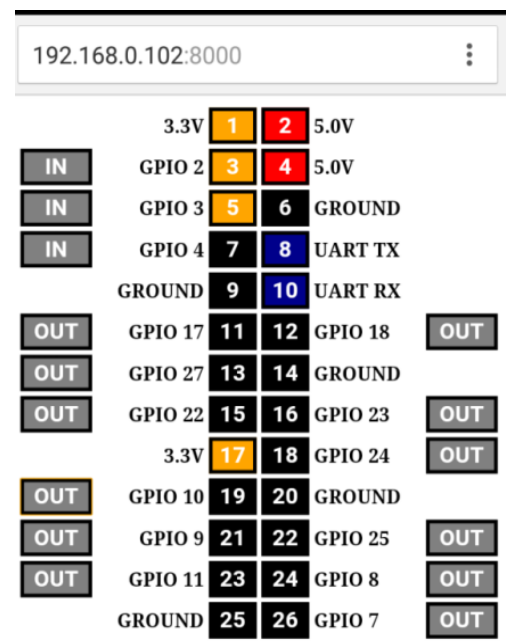

Fig.3: GPIO Header file of Raspberry Pi

The IN and OUT pins of the GPIO are mentioned, shows the current status of the appliances.8 GPIO pins for external peripheral connections supported by Raspberry Pi. 2- 3.3V, 2-5V pins for power supply connected to the external devices. With the Python is a default programming language for the Raspberry Pi with support of C, C++, Java, Perl and Ruby.

2.3.1 LAN Cable (Local Area Network)

To access the internet in Raspberry Pi we need LAN connection, with the help of which we can access the incoming and outgoing E- mail services. The LAN speed is much faster than the wireless connection.

\subsubsection{HDMI Display}

To see the current status of the home appliances as well as the sensors (LPG gas, PIR, temperature) and also we will able to check the updates regarding Raspberry Pi.

\subsubsection{PIR sensor}

Passive Infrared sensor is to detect the motion of human being, as a human passes through this sensor, the temperature in the background will rise from room temperature to the body temperature and thus the motion or human will be detected.

\subsubsection{Buzzer}

It has two wires. Namely red black. Polarity matters: black=ground Apply an oscillating voltage to make a noise. Oscillating voltage alternately squeezes and releases the piezo element. Must apply flucuating voltage, a steady HIGH or LOW won't work. The buzzer case supports the piezo element and has resonant cavity for sound

\subsubsection{Keyboard and mouse}

Key board and mouse are used to operate the Raspberry Pi, so we can easily do the programming and make changes easily.

\subsubsection{Webcam}

A webcam is a mini videocamera that has the capability to feeds or stream its image in real time to, or through a computer to a computer network. When the image is "captured" by the computer, the video stream is saved, viewed or sent on to other networks via a systems such as internet, and email as an attachment. When sent to a remote location, the video stream may be saved, viewed or sent there. Unlike an IP camera (which connects using Ethernet or Wi-Fi), a webcam is generally connected by a USB cable, or similar cable, or built into the computer hardware, such as laptops.

\subsection{INTERFACING}

- First of all need to install the Rasbian Operating system in the micro SD card, after that need to insert the SD card in Raspberry Pi kit and give the 5V power supply to the same. 


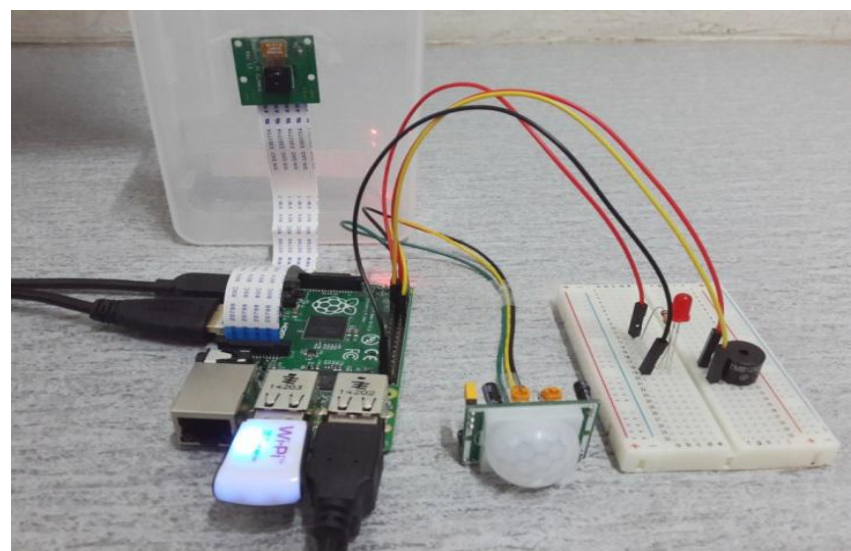

Fig.4: Motion detecting camera security system experimental setup

- Connect the HDMI port to the LED TV and thus the display will be observed on LED screen.

- Now connect the keyboard and mouse to the Raspberry Pi USB ports, so will be able to write the code with the help of keyboard and mouse.

- Connect the LAN cable to Ethernet port and so we can access the Raspberry Pi and make the system interactive by sending and receiving E-mails.

- Need to write the code for the proposed system in Python language and store the results in the form of Email.

- LEDs are used to check the hardware setup before the interfacing and it will show either the device is in ON state or OFF state.

\section{RESULTS}

\subsection{CASE I}

When the email has been successfully sent, the LED and buzzer will 'OFF' and stop producing alarm sound respectively.

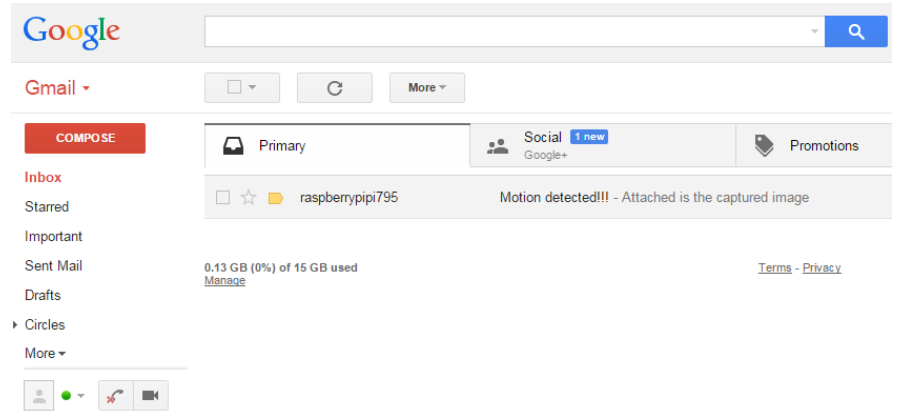

Fig.5: Email notification

3.2 CASE II

Figure 6 show the notification and content of the email received by the user respectively.

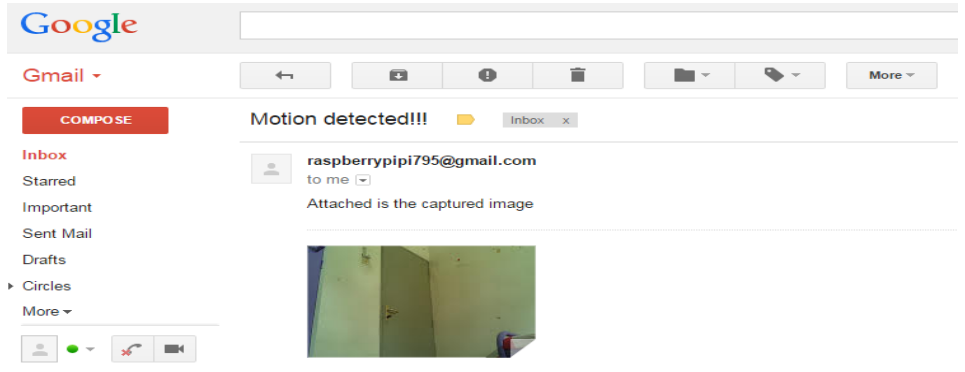

\subsection{CASE III}

Fig.6: Content of the received email 
The main purpose of this android application was to enable the user to remotely control the activation of the system. 'Taken image' button is used to view the captured image once a motion is detected. Therefore the user can further confirm the reliability of the received image from the email.

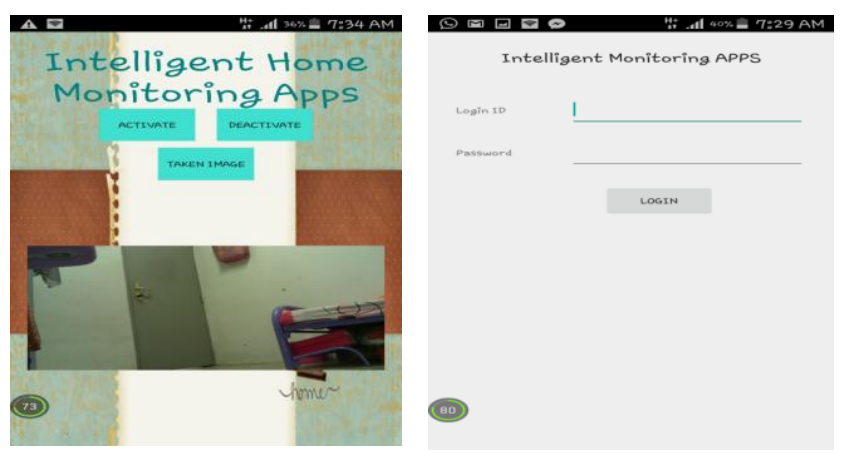

Fig.7: Android application interface

\subsection{CASE IV}

The home surveillance system is then integrated with the Android application to develop an intelligent motion detecting camera security system for home/offices. The complete Python program for the motion detecting camera security system. The system will only be activated when the user touches the 'Activate' button. Once the 'Activate' button is touched, the motion detecting camera security will performed their complete function. Otherwise, the user can deactivate the system by touching the 'Deactivate' button. Figure 6.7 shows the result obtained from the system integration.

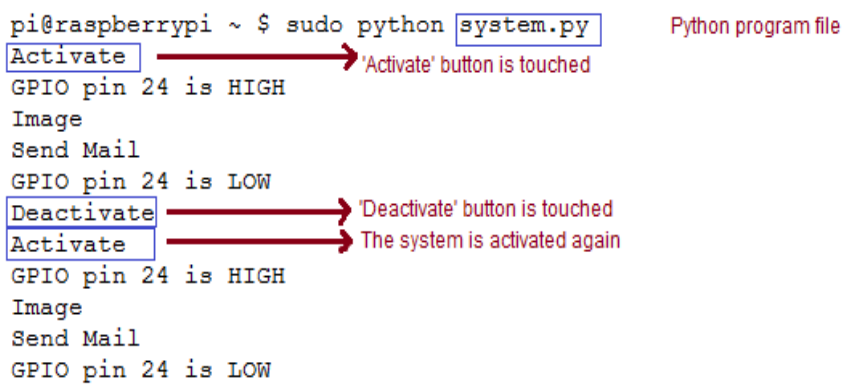

Fig.8: Result of the system integration

\section{CONCLUSION}

We have developed a comprehensive solution that can provides a user friendly home based automation and security application for all homes/offices. We accomplished this project through the integration of cheap, off-the-shelf, widely available devices, interfaces and software coupled with a user friendly interface. This work provides users with an easy to use mobile application for which they can remotely access and control their home appliances and security. In future we intend to provide a wireless relay connection and wireless sensors which can be movable and can be operated and which can be used in company and instates for Security to the whole building with one single system. This provides a full security support for homes.

\section{REFERENCES}

[1] Sharma, Rupam Kumar, et al. "Android interface based GSM home security system", Issues and Challenges in Intelligent Computing Techniques (ICICT), Proc. IEEE Conf., 2014.

[2] D. Luca, Gabriele, et al. "The use of NFC and Android technologies to enable a KNX-based smart home", Software, Telecommunications and Computer Networks (SoftCOM), Proc. $21^{\text {st }}$ IEEE Conf., 2013.

[3] Gu, Yi, et al. "Design and Implementation of UPnP-Based Surveillance Camera System for Home Security", Information Science and Applications (ICISA), Proc. IEEE Conf., 2013. 
[4] Van Thanh Trung, Bui, and Nguyen Van Cuong. "Monitoring and controlling devices system by GPRS on FPGA platform", Advanced Technologies for Communications (ATC), Proc. IEEE Conf., 2013.

[5] Karia, Deepak, et al. "Performance analysis of ZigBee based Load Control and power monitoring system", Advances in Computing, Communications and Informatics (ICACCI), Proc. IEEE Conf., 2013.

[6] Ryu, Yeonghyeon, Jeakyu Yoo, and Youngroc Kim. "Cloud services based Mobile monitoring for Photovoltaic Systems", Cloud Computing Technology and Science (CloudCom), Proc. IEEE Conf., 2012.

[7] Robson, Clyde, et al. "High performance web applications for secure system monitoring and control", Proc. IEEE Conf., Nuclear Science Symposium and Medical Imaging Conference (NSS/MIC), 2012.

[8] Han, Jinsoo, et al. "User-friendly home automation based on 3D virtual world", IEEE Trans. Consumer Electronics, 56(3), 2010, 18431847.

[9] Xu, Lingshan, et al. "A Cloud-based monitoring framework for Smart Home."Cloud Computing Technology and Science (CloudCom), Proc. $4^{\text {th }}$ IEEE Conf., 2012.

[10] Bajorek, Marcin, and Jedrzej Nowak. "The role of a mobile device in a home monitoring healthcare system." Computer Science and Information Systems (FedCSIS), Proc. Federated IEEE Conf., 2011. 